\title{
Community-Based Group Physical Activity and/or Nutrition Interventions to Promote Health and Mobility in Older Adults: An Umbrella Review
}

Sarah Neil-Sztramko ( $\nabla$ neilszts@mcmaster.ca )

McMaster University

Kylie Teggart

McMaster University

Caroline Moore

McMaster University

Diana Sherifali

McMaster University

Donna Fitzpatrick-Lewis

McMaster University

Giulia Coletta

McMaster University

Stuart Phillips

McMaster University

K Bruce Newbold

McMaster University

Elizabeth Alvarez

McMaster University

Ayse Kuspinar

McMaster University

Courtney Kennedy

McMaster University

Lina Santaguida

McMaster University

Rebecca Ganann

McMaster University

\section{Research Article}

Keywords: mobility, nutrition, older adults, physical activity, umbrella review 
Posted Date: September 9th, 2021

DOI: https://doi.org/10.21203/rs.3.rs-578194/v2

License: (c) (i) This work is licensed under a Creative Commons Attribution 4.0 International License. Read Full License 


\section{Abstract \\ Background}

Physical activity and a healthy diet are important in helping to maintain mobility and quality of life with aging. Delivery of physical activity and nutrition interventions in a group setting adds the benefits of social participation.

\section{Objectives}

This umbrella review aims to identify group-based physical activity and nutrition interventions for community-dwelling older adults that improve mobility.

\section{Methods}

Five electronic databases (MEDLINE, Embase, CINAHL, Cochrane CENTRAL, Sociological Abstracts) were searched from inception to April 28, 2020. Eligibility criteria included systematic reviews exploring the effectiveness of physical activity and/or nutrition interventions, delivered in a group setting for community-dwelling older adults. Two reviewers independently performed eligibility screening, critical appraisal (using AMSTAR 2) and data extraction. Older adult/provider research partners informed data synthesis and results presentation.

\section{Results}

In total, 54 systematic reviews (1 high, 21 moderate, 32 low/critically low quality) were identified; 46 included physical activity only, and eight included both physical activity and nutritional supplements. No reviews included nutrition interventions alone. Combined aerobic/resistance, general physical activity, and mind-body exercise all improved physical function and balance (moderate-high certainty). Aerobic/resistance training improved aerobic capacity (high certainty). Resistance training and general physical activity improved muscle strength (moderate certainty). Aerobic/resistance training and general physical activity are likely to reduce falls among older adults (moderate certainty). There was no evidence of benefit for nutritional supplementation with physical activity.

\section{Conclusions}

Multicomponent group-based physical activity interventions can improve measures of mobility in community-dwelling older adults. We found no reviews focused on nutrition only, highlighting a gap in the literature. 


\section{Background}

Mobility is a multifaceted construct, influenced by a range of physical, cognitive, psychosocial, environmental, financial, gender, cultural, and biographical factors (1). A comprehensive view of mobility reflects one's ability to move within their immediate home environment and the broader community (1). Reductions in mobility and the ability to carry out activities of daily living are common with aging and are recognized precursors to frailty, hospitalization, and death $(2,3)$. The effect of physical activity (PA) and diet quality on physical, cognitive, and social outcomes has been widely demonstrated; however, inactivity and malnutrition continue to affect the well-being and mobility of older adults (4-8).

Community-based programs delivered in group settings can address both the physical and psychosocial aspects of mobility, promoting a sense of belonging which aids in long-term adherence $(9,10)$. The Enhancing physical and community MoBility in OLDEr adults with health inequities using commuNity codesign (EMBOLDEN) trial is a multi-year program of research from XX University in [City, Country] (11). EMBOLDEN uses community-based co-design to integrate local community needs, preferences, and resources with high-quality scientific evidence. The interdisciplinary team of researchers collaborates with older adults and community organizations to enhance personal mobility and address health inequities.

Several systematic reviews have been published exploring a broad range of PA and/or nutrition interventions for older adults, making it challenging to bring together the best scientific evidence to inform program design. Umbrella reviews provide a rigorous methodology for synthesizing evidence from multiple existing systematic reviews (12), and may be particularly useful for a phenomenon such as mobility given the wide variety of interventions and uncertainty as to which interventions are more effective when delivered individually or in combination and within different populations and/or settings. To date, two umbrella reviews have reported the effectiveness of exercise interventions in pre-frail, frail, or sarcopenic older adults $(13,14)$, and one umbrella review has described the impact of nutritional interventions for community-dwelling older adults on body composition (15). Given the lack of recent, relevant synthesized evidence to meet our needs, our team undertook this umbrella review to help inform intervention design and provide a foundation for the EMBOLDEN project. This umbrella review aims to synthesize evidence from existing systematic reviews regarding the effectiveness of group-based PA and/or nutrition interventions to improve mobility in community-dwelling older adults.

\section{Methods}

This review was conducted following the Joanna Briggs Institute (JBI) guidance for umbrella reviews (12), and was registered with PROSPERO (CRD42020141352). Although originally conceptualized as a systematic review, upon initiation of screening it was determined that many systematic reviews existed, and an umbrella review was most appropriate.

\section{Search Strategy}


A trained librarian conducted a search of MEDLINE, Embase, CINAHL, Cochrane CENTRAL, and Sociological Abstracts from inception to April 28, 2020 (Additional file 1). Searches were limited to systematic reviews/meta-analyses and randomized controlled trials (RCTs) published in English.

\section{Study Selection}

Citations were imported into DistillerSR (Evidence Partners, Ottawa, Canada) and duplicates were removed. Citations were reviewed by two independent reviewers using pre-determined criteria. At the title/abstract level, a study must have been selected by one reviewer for inclusion, while exclusion required two reviewers to agree. At the full-text level, disagreements were resolved through discussion by two reviewers, with input from a third team member as needed.

\section{Eligibility Criteria \\ Types of Studies}

Systematic reviews (narrative summary, meta-analysis, or network meta-analysis) of interventions published since 2010 were eligible. Scoping or narrative reviews that did not include critical appraisal of primary studies were excluded. Eligible reviews could include RCTs and non-randomized intervention studies, however, at least $80 \%$ of single studies included must have been interventions (i.e., not descriptive, qualitative, or observational).

\section{Types of Participants}

Eligible systematic reviews included studies involving community-dwelling older adults. Reviews were included if the pooled mean age or inclusion criteria identified an age of $\geq 55$ years. If this information was not available, at least $70 \%$ of included studies must have reported a mean sample age of $\geq 55$ years. Reviews in which studies were selected based on a specific health or disease status (e.g., cancer, sarcopenia) were excluded. In reviews that did not restrict by disease status, $70 \%$ of included studies must have been conducted in general sample of older adults. The choice of $70 \%$ was intended to include reviews in which the majority of included studies were relevant to the general population; most studies were either well above or well below this threshold.

\section{Interventions}

Eligible reviews must have included single studies of any PA (any movement resulting in energy expenditure), structured exercise (planned and repetitive movements), and/or nutrition intervention that could reasonably be delivered in a group-based setting (16).

\section{Context}

Single studies within eligible reviews must have been delivered in a community setting. Reviews that focused exclusively on interventions delivered in hospitals, rehabilitation centers, long-term care homes, or clinics were excluded. When the reviews did not set inclusion criteria by setting, at least $70 \%$ of 


\section{Outcomes}

Reviews must have synthesized (narratively or via meta-analysis) outcomes related to physical or community mobility. These outcomes were broadly classified into six domains: aerobic capacity, physical function, balance, falls/safety, muscular strength, and self-reported mental wellbeing/quality of life. Reviews that focused exclusively on cognitive function or body composition were excluded. These criteria were not part of our original protocol as registered in PROSPERO but added at the full text screening level as the goals of these interventions and associated outcomes were quite distinct.

\section{Assessment of Methodological Quality}

Eligible reviews were critically appraised using A MeaSurement Tool to Assess systematic Reviews (AMSTAR 2) (17). AMSTAR 2 was completed independently by two reviewers, with conflicts resolved through discussion or the input of a third reviewer, as needed. Following consensus, results were entered into the online AMSTAR checklist, which provides an assessment of overall quality as critically low, low, moderate, or high based on seven critical domains (18).

\section{Data Extraction}

Data were extracted by two independent reviewers using a standardized form. Disagreements were resolved through discussion or by a third reviewer. Data were extracted related to review methodology (e.g., sources searched, publication date range, methodological quality of included studies, noted limitations) and details of included studies (e.g., study designs, participant characteristics, intervention descriptions, setting). To explore issues of equity, diversity and inclusion, any data regarding material deprivation, and percentage of low-income and/or immigrant populations were also extracted. Results from both narrative syntheses and meta-analyses were extracted within the six outcome categories described above. Any outcomes within these categories or composite outcomes in these areas (e.g., when multiple outcome measures were grouped and reported as standardized mean difference in a metaanalysis) were extracted, as reported. Data collection forms and full extracted data are available upon request.

\section{Data Synthesis and Certainty of Evidence}

The Grading of Recommendations Assessment, Development and Evaluation (GRADE) approach was used to assess the overall certainty of the evidence (19). The GRADE process was adapted to accommodate the umbrella review by considering both the findings across included reviews and across single studies within reviews by intervention type and outcome. Following the GRADE approach, reviews including primarily RCTs start at 'high' certainty, while reviews primarily including non-randomized studies start at 'low' certainty. The level of certainty was further downgraded based on risk of bias, inconsistency of findings, indirectness of interventions/outcomes, imprecision of effect measures, and/or publication bias, and were upgraded based on magnitude of effect size, dose-response relationship, and accounting for confounding. A narrative approach to data synthesis was used, with results presented in supporting 
size of the effect are presented to communicate overall findings within each intervention type and outcome category, in line with published recommendations (20). Only results that compared an intervention group to a control group were included in GRADE, although subgroup analyses are presented in accompanying tables.

The review team synthesized data with feedback and input from the larger research team and key stakeholders. After an initial draft, preliminary results and categories were presented to four older adult citizen and service provider partners from an established stakeholder group within the EMBOLDEN research program who were consulted via a one-time, virtual meeting. The aim of this engagement was to allow for feedback (via discussion) about the appropriateness of intervention/outcome groupings and to identify priority outcome measures (e.g., prioritize general physical function outcomes over measures such as body composition). Our older adult and service provider partners also contributed to developing public-facing documents, including a plain-language summary and infographic.

\section{Results}

The search identified 36,535 unique citations, of which 1,108 were potentially relevant; at this point, the team elected to limit to systematic reviews (Figure 1). A second screen identified 337 citations for full-text review, of which 54 were included (Table 1). A list of excluded studies with reasons is provided in Additional file 2.

Included reviews reported on several types of interventions, with some reporting separate results for more than one intervention type. Most reviews focused on exercise or PA only $(n=46)(21-66)$, while others included exercise with nutritional supplements $(n=8)(67-74)$. No reviews included group-based nutrition interventions alone. Exercise or PA interventions were categorized as resistance exercise $(n=11)(21,30$, $34,35,38,46,56,61-63,75)$, aerobic exercise $(n=4)(22,24,28,51)$, combined aerobic and resistance exercise $(n=9)(23,29,34,36,44,46,50,63,75)$, general PA $(n=9)(32,33,35,40,45,49,52,54,66)$, mind-body exercise (e.g., Tai Chi, yoga, Pilates) $(n=8)(26,27,35,43,47,55,58,59)$, dance $(n=5)(31,37$, $48,57,63)$, and other $(n=10)(25,34,35,39,42,53,63-65,76)$. Nutritional supplements included protein $(n=4)(69,72-74)$, creatine $(n=2)(67,71)$, vitamin $D(n=1)(68)$, or dairy $(n=1)(70)$. Meta-analyses were undertaken in 34 reviews $(21,22,26-30,32-35,40,42,43,46,48,51,53,54,56,59,61-63,65,66,68$, $70-75,77), 19$ reviews presented findings narratively $(23,24,31,37-39,44,45,47,49,50,52,55,57,58,64$, $67,69,76)$, and one performed a network meta-analysis (25). Total sample sizes ranged from 153-28,523 when reported. Participants ranged from 42-98 years old, with most reviews only including studies with participants aged 60 and older. No reviews extracted data on material deprivation, low income, or immigrant populations.

Eligible reviews included 1,170 primary studies, of which 853 were unique ( $27.1 \%$ overlap across reviews, although some duplicates were included in reviews focused on different intervention types). Reviews with the most overlap by intervention type were exercise with nutritional supplements (36.2\% overlap), dance (21 a\% nvarlan) and rocictanno ovornico $/ 26$ 7\% nvarlan) Single studies were published between 1983Loading [MathJax]/jax/output/CommonHTML/fonts/TeX/fontdata.js 
2020 (range 5 to 99 studies per review). Of these, $82 \%$ were randomized controlled trials and $18 \%$ were quasi-experimental, observational, or not reported.

\section{Methodological Quality of Included Reviews}

Methodological quality of the reviews was variable (summary in Figure 2, full assessment in Additional file 3), with one review (35) rated as having high confidence in findings. The confidence for the remaining reviews were moderate $(n=21)(24,25,30,32,33,39,51,52,55,56,59,62-65,68-71,76,77)$, low $(n=$ 14) $(26,29,31,40,42,43,46,49,54,57,58,66,67,72)$, and critically low $(n=18)(21-23,27,28,34,37$, $38,44,45,47,48,50,53,61,73-75)$. Most reviews did not report protocol registration, describe an adequate search strategy, justify excluded studies, or incorporate risk of bias in interpreting review findings.

\section{Findings of Reviews}

A summary of findings by intervention type and outcome category, alongside review quality is listed in Table 2, with summary of certainty of evidence (GRADE) in Figure 3.

\section{Aerobic capacity}

The effect of interventions on aerobic capacity was reported in 13 reviews (Additional file 4). Across reviews, 97 studies were reported, of which 92 were unique (overlap, 5.2\% across intervention types). Aerobic capacity was most often assessed using measured or predicted maximal or peak oxygen consumption ( $\mathrm{VO}_{2}$ peak). Based on high-certainty evidence, a combination of aerobic and resistance training results in meaningful improvements in aerobic capacity in older adults. Based on moderate certainty evidence, aerobic exercise probably results in improvements in aerobic capacity. Dance interventions may result in increased aerobic capacity, although this is based on low-certainty evidence and findings may change as more information becomes available. Interventions that combined exercise with nutritional supplements may make little to no difference in aerobic capacity of older adults, although this is based on low-certainty evidence. The evidence is very uncertain about the effect of mind-body exercise or resistance exercise alone on aerobic capacity in older adults (very low certainty evidence). Other exercise types, including aquatic exercise and a combination of PA and cognitive training were also examined for their effect on aerobic capacity. No included reviews explored the effect of general PA interventions on aerobic capacity.

\section{Physical function}

In total, 44 reviews reported on 516 single studies, of which 372 were unique ( $27.9 \%$ overlap across intervention types). Physical function was typically assessed using the Timed Up and Go test, chair stands, gait speed, and six-minute walk test; often findings from single studies were compiled into a composite score for self-reported and/or measured physical function within meta-analyses (Additional file 5). Based on moderate certainty evidence, interventions that included a combination of aerobic and 
resistance training, interventions focused on general PA, and mind-body exercise interventions are all likely to result in improvements in physical function in older adults. Resistance training and dance interventions may also increase physical function (low certainty evidence). Low certainty evidence suggests that aerobic exercise interventions and exercise combined with nutritional supplements may have little to no impact on physical function.

\section{Balance}

In total, 25 reviews reported on 217 single studies, of which 181 were unique (16.6\% overlap). Static and dynamic balance tests (e.g., single-leg stance, Berg Balance Scale) and composite balance measures were used across reviews (Additional file 6). High certainty evidence suggests that participation in mindbody exercise interventions increases balance in older adults. General PA interventions and interventions that combined aerobic and resistance training are also likely to result in improvements in balance in older adults, based on moderate-certainty evidence. Dance interventions may improve balance; however, this is based on low certainty evidence. Also based on low certainty evidence, resistance training and aerobic exercise alone may result in little to no change in balance. No included reviews explored the effects of exercise and nutritional supplements on balance.

\section{Falls and safety}

Number of falls, risk of falling, and fall-related injuries were measured across 10 reviews including 88 single studies, 78 of which were unique (11.4\% overlap). Interventions that combined aerobic and resistance exercise and interventions focused on general PA are likely to result in a small reduction in the risk of falls or fall-related injuries in older adults, based on moderate certainty evidence (Additional file 7). Based on low-certainty evidence, mind-body exercises may have little to no meaningful effect on fall risk, although these findings may change as more data are available. Dance interventions may reduce falls, but the evidence is of very low certainty. Also based on very low certainty evidence, aerobic training alone and resistance training alone may have little to no effect on falls risk. No reviews reported the risk of falls within interventions that combined exercise and nutrition.

\section{Muscle strength}

Within reviews reporting muscle strength outcomes, 34 reviews reported on 369 single studies, of which 292 were unique (20.9\% overlap). Various measures were reported, including handgrip strength, upper body strength, lower body strength, muscle mass, and overall muscle strength (Additional file 8). Both resistance exercise interventions and general PA interventions likely increase upper and lower body strength (moderate certainty evidence). Aerobic exercise alone, combined aerobic and resistance exercise, mind-body exercise, and dance interventions may result in improvements in muscle strength, however this is based on low certainty evidence and findings may change as more data become available. Also based on low certainty evidence, interventions that combined exercise with nutritional supplements may not improve muscle strength. 
In total, 14 reviews reported health-related quality of life and self-reported wellbeing outcomes (Additional file 9). Given the variation in constructs measured within this domain (e.g., activities of daily living, quality of life (SF-36), perceived mental health) and limited number of reviews for each outcome type, these results were not incorporated into the overall summary of findings using GRADE.

\section{Discussion}

We provide a high-level synthesis regarding overall effectiveness of group-based PA and nutrition interventions to improve mobility among community-dwelling older adults. Interventions that combined aerobic and resistance exercise, and general PA interventions were found to result in meaningful improvements in physical function, balance, aerobic capacity, and muscle strength in older adults, and are also likely to reduce falls and fall-related injuries.

Our findings support a multifaceted approach to health and wellbeing among community-dwelling older adults. Similar findings are reflected in two overviews of reviews focused on all adults over the age of 18 , including older adults $(78,79)$, which informed the recent Canadian 24-Hour Movement Guidelines (80). These guidelines also recommend a combination of aerobic, resistance, and balance exercises for adults aged 65+. Recent evidence has found that older adults face unique barriers and hesitancy to engage in certain types of exercise, such as resistance training (81). It is encouraging that benefits for each of our outcome domains were seen across a range of intervention types. This suggests that effective interventions for older adults can incorporate a variety of type of exercises or physical activities that are most likely foster enjoyment. This approach can also improve accessibility to PA within this population by building upon existing community services and group-based PA programs that provide the additional benefit of social participation, which enhances enjoyment, adherence, and sustainability of PA (82).

Our findings did not provide any convincing evidence for the addition of protein, creatine, vitamin $D$, or dairy supplementation to PA interventions within community-dwelling older adults. However, the overall quality of the systematic reviews and single studies was low to moderate, and numerous distinct comparator groups were used to test intervention effectiveness. These reviews typically synthesized highly heterogeneous single studies, including a wide range in "dose" of both exercise and dietary components of the interventions; this may have limited the ability to see effects of specific combinations of interventions when synthesized together. Future high-quality studies with similar intervention and comparator groups may provide better understanding of the role of combined diet and nutrition interventions on mobility-related outcomes in older adults. No reviews focused on group-based nutrition interventions alone, nor did any explore or report on domains of equity, diversity, and inclusion, highlighting priorities for future research.

There are several inherent limitations of this umbrella review that should be considered in interpreting results. Included reviews were limited to those in English, published since 2010. At the systematic review 
collect this data. Given the large number of included reviews, the overlap in single studies across reviews is unsurprising. The highest amount of overlap of studies evaluating physical function outcomes is attributable to our broad characterization of this outcome and the overlap in single studies among reviews focused on resistance, exercise and nutrition, and dance interventions. Although $27.9 \%$ overlap in single studies exists, each review contributing to these results focused on specific outcomes (e.g., gait speed alone, composite physical function measures), and we do not anticipate this greatly influenced our overall certainty of evidence. Although we would expect targeted aerobic, resistance, or combined aerobic and resistance exercise to be more effective than general PA interventions, certainty in the evidence was influenced by higher risk of bias and heterogeneity across both single studies and reviews of aerobic, resistance and combined interventions, reflective of variation in types of interventions and tools used to assess outcomes.

A strength of this umbrella review was the collaboration with older adults and service provider partners to identify relevant outcomes. Our partners prioritized the inclusion of quality of life and wellbeing outcomes, as these outcomes are front of mind for older adults and providers. Self-reported functional measures were found to be more meaningful to our older adult partners than measures designed to capture physiology or function. However, very few studies reported these outcomes separately as they were commonly combined within meta-analyses, thus we are unable to distinguish between self-reported and measured function.

\section{Conclusion}

Group and community-based PA interventions that combine aerobic and resistance, general PA and mindbody exercise can improve mobility measures in older adults. No reviews focused on group-based nutrition interventions alone, and very few identified quality of life outcomes, highlighting a need for future synthesis work. The results of this umbrella review will be used to inform the co-design of a community-based, mobility-enhancing intervention.

\section{Abbreviations}

AMSTAR 2: A MeaSurement Tool to Assess systematic Reviews

EMBOLDEN: Enhancing physical and community MoBility in OLDEr adults with health inequities using commuNity co-design trial

GRADE: Grading of Recommendations Assessment, Development and Evaluation

JBI: Joanna Briggs Institute

PA: physical activity

RCT: randomized controlled trial 


\section{Declarations}

\section{Ethics approval and consent to participate}

Not applicable

\section{Consent for publication}

Not applicable

\section{Availability of data and materials}

The datasets supporting the conclusions of this article are included within the article and its additional files.

\section{Competing interests}

SMP declares that he is a named inventor on a patent held by Exerkine, but receives no fees/payment, and is an unpaid member of the Scientific Advisory Board for Enhanced Recovery.

\section{Funding}

Funding received from the Labarge Centre for Mobility in Aging within the McMaster Institute for Research on Aging at McMaster University, Canada Research Chairs Program, the Canadian Institutes of Health Research, and in-kind support from the Aging, Community and Health Research Unit at McMaster University. The funders had no role in the study design, conduct, or decision to publish.

\section{Authors' contributions}

SNS, CM, DS, DFL, SMP, KBN, EA, AK, CCK, PLS, and RG conceptualized and designed the study. SNS, KT, $\mathrm{CM}, \mathrm{DS}, \mathrm{DFL}, \mathrm{GC}$, and RG acquired, analyzed, and interpreted the data. SNS, KT, and RG drafted the manuscript. All authors substantively revised the manuscript and have approved the submitted version.

\section{Acknowledgments}

Not applicable

\section{References}

1. Webber SC, Porter MM, Menec VH. Mobility in older adults: a comprehensive framework. Gerontologist. 2010;50(4):443-50.

2. Tuscany Regional Health Council. Frailty in elderly people 2013 [cited 2021 May]. Available from: https://www.regione.toscana.it/documents/10180/320308/Frailty+in+elderly+people/9327bb856d3c-4e1b-a398-669e76ce5b01?version=1.0. 
3. Wen YC, Chen LK, Hsiao FY. Predicting mortality and hospitalization of older adults by the multimorbidity frailty index. PLoS One. 2017;12(11):e0187825.

4. World Health Organization. World Report on Ageing and Health Geneva, Switzerland: World Health Organization; 2015 [cited 2021 May]. Available from: https://apps.who.int/iris/handle/10665/186463.

5. Ramage-Morin PL, Garriguet D. Nutritional risk among older Canadians. Health Rep. 2013;24(3):313.

6. Bandayrel K, Wong S. Systematic literature review of randomized control trials assessing the effectiveness of nutrition interventions in community-dwelling older adults. J Nutr Educ Behav. 2011;43(4):251-62.

7. Pahor M, Guralnik JM, Ambrosius WT, Blair S, Bonds DE, Church TS, et al. Effect of structured physical activity on prevention of major mobility disability in older adults: the LIFE study randomized clinical trial. JAMA. 2014;311(23):2387-96.

8. Young K, Bunn F, Trivedi D, Dickinson A. Nutritional education for community dwelling older people: a systematic review of randomised controlled trials. Int J Nurs Stud. 2011;48(6):751-80.

9. Landi F, Cesari M, Calvani R, Cherubini A, Di Bari M, Bejuit R, et al. The "Sarcopenia and Physical fRailty IN older people: multi-componenT Treatment strategies" (SPRINTT) randomized controlled trial: design and methods. Aging Clin Exp Res. 2017;29(1):89-100.

10. Farrance C, Tsofliou F, Clark C. Adherence to community based group exercise interventions for older people: A mixed-methods systematic review. Prev Med. 2016;87:155-66.

11. Neil-Sztramko SE, Teggart K, Phillips S, Sherifali D, Fitzpatrick-Lewis D, Newbold B, et al. An evidenceinformed and stakeholder co-designed physical activity and community mobility intervention for older adults facing health inequities: the EMBOLDEN study. Advances in Evidence Synthesis: special issue. Cochrane Database of Systematic Reviews. 2020;9:21.

12. Aromataris E, Fernandez R, Godfrey C, Holly C, Khalil H, Tungpunkom P. Chapter 10: Umbrella Reviews. JBI Manual for Evidence Synthesis 2020 [cited 2021 May]. Available from: https://doi.org/10.46658/JBIMES-20-11.

13. Jadczak AD, Makwana N, Luscombe-Marsh N, Visvanathan R, Schultz TJ. Effectiveness of exercise interventions on physical function in community-dwelling frail older people: an umbrella review of systematic reviews. JBI Database System Rev Implement Rep. 2018;16(3):752-75.

14. Moore SA, Hrisos N, Errington L, Rochester L, Rodgers $H$, Witham M, et al. Exercise as a treatment for sarcopenia: an umbrella review of systematic review evidence. Physiotherapy. 2020;107:189-201.

15. Schultz TJ, Roupas P, Wiechula R, Krause D, Gravier S, Tuckett A, et al. Nutritional interventions for optimizing healthy body composition in older adults in the community: an umbrella review of systematic reviews. JBI Database System Rev Implement Rep. 2016;14(8):257-308.

16. Caspersen CJ, Powell KE, Christenson GM. Physical activity, exercise, and physical fitness: definitions and distinctions for health-related research. Public Health Rep. 1985;100(2):126-31. 
17. Shea BJ, Reeves BC, Wells G, Thuku M, Hamel C, Moran J, et al. AMSTAR 2: a critical appraisal tool for systematic reviews that include randomised or non-randomised studies of healthcare interventions, or both. BMJ. 2017;358:j4008.

18. Shea BJ, Reeves BC, Wells G, Thuku M, Hamel C, Moran J, et al. AMSTAR Checklist 2017 [cited 2021 May]. Available from: https://amstar.ca/Amstar_Checklist.php.

19. Guyatt GH, Oxman AD, Vist GE, Kunz R, Falck-Ytter Y, Alonso-Coello P, et al. GRADE: an emerging consensus on rating quality of evidence and strength of recommendations. BMJ. 2008;336(7650):924-6.

20. Santesso N, Glenton C, Dahm P, Garner P, Akl EA, Alper B, et al. GRADE guidelines 26: informative statements to communicate the findings of systematic reviews of interventions. Journal of Clinical Epidemiology. 2020;119:126-35.

21. Borde R, Hortobagyi T, Granacher U. Dose-Response Relationships of Resistance Training in Healthy Old Adults: A Systematic Review and Meta-Analysis. Sports Med. 2015;45(12):1693-720.

22. Bouaziz W, Kanagaratnam L, Vogel T, Schmitt E, Drame M, Kaltenbach G, et al. Effect of Aerobic Training on Peak Oxygen Uptake Among Seniors Aged 70 or Older: A Meta-Analysis of Randomized Controlled Trials. Rejuvenation Res. 2018;21(4):341-9.

23. Bouaziz W, Lang PO, Schmitt E, Kaltenbach G, Geny B, Vogel T. Health benefits of multicomponent training programmes in seniors: a systematic review. Int J Clin Pract. 2016;70(7):520-36.

24. Bouaziz W, Vogel T, Schmitt E, Kaltenbach G, Geny B, Lang PO. Health benefits of aerobic training programs in adults aged 70 and over: a systematic review. Arch Gerontol Geriatr. 2017;69:110-27.

25. Bruderer-Hofstetter M, Rausch-Osthoff AK, Meichtry A, Munzer T, Niedermann K. Effective multicomponent interventions in comparison to active control and no interventions on physical capacity, cognitive function and instrumental activities of daily living in elderly people with and without mild impaired cognition - A systematic review and network meta-analysis. Ageing Res Rev. 2018;45:1-14.

26. Bueno de Souza RO, Marcon LF, Arruda ASF, Pontes Junior FL, Melo RC. Effects of Mat Pilates on Physical Functional Performance of Older Adults: A Meta-analysis of Randomized Controlled Trials. Am J Phys Med Rehabil. 2018;97(6):414-25.

27. Bullo V, Bergamin M, Gobbo S, Sieverdes JC, Zaccaria M, Neunhaeuserer D, et al. The effects of Pilates exercise training on physical fitness and wellbeing in the elderly: A systematic review for future exercise prescription. Prev Med. 2015;75:1-11.

28. Bullo V, Gobbo S, Vendramin B, Duregon F, Cugusi L, Di Blasio A, et al. Nordic Walking Can Be Incorporated in the Exercise Prescription to Increase Aerobic Capacity, Strength, and Quality of Life for Elderly: A Systematic Review and Meta-Analysis. Rejuvenation Res. 2018;21(2):141-61.

29. Chase JD, Phillips LJ, Brown M. Physical Activity Intervention Effects on Physical Function Among Community-Dwelling Older Adults: A Systematic Review and Meta-Analysis. J Aging Phys Act. 2017;25(1):149-70. 
30. da Rosa Orssatto LB, de la Rocha Freitas C, Shield AJ, Silveira Pinto R, Trajano GS. Effects of resistance training concentric velocity on older adults' functional capacity: A systematic review and meta-analysis of randomised trials. Exp Gerontol. 2019;127:110731.

31. Fernandez-Arguelles EL, Rodriguez-Mansilla J, Antunez LE, Garrido-Ardila EM, Munoz RP. Effects of dancing on the risk of falling related factors of healthy older adults: a systematic review. Arch Gerontol Geriatr. 2015;60(1):1-8.

32. Frost R, Belk C, Jovicic A, Ricciardi F, Kharicha K, Gardner B, et al. Health promotion interventions for community-dwelling older people with mild or pre-frailty: a systematic review and meta-analysis. BMC Geriatr. 2017;17(1):157.

33. Garcia-Hermoso A, Ramirez-Velez R, Saez de Asteasu ML, Martinez-Velilla N, Zambom-Ferraresi F, Valenzuela PL, et al. Safety and Effectiveness of Long-Term Exercise Interventions in Older Adults: A Systematic Review and Meta-analysis of Randomized Controlled Trials. Sports Med. 2020;50(6):1095-106.

34. Hortobagyi T, Lesinski M, Gabler M, VanSwearingen JM, Malatesta D, Granacher U. Effects of Three Types of Exercise Interventions on Healthy Old Adults' Gait Speed: A Systematic Review and MetaAnalysis. Sports Med. 2015;45(12):1627-43.

35. Howe TE, Rochester L, Neil F, Skelton DA, Ballinger C. Exercise for improving balance in older people. Cochrane Database Syst Rev. 2011(11):CD004963.

36. Hurst C, Weston KL, McLaren SJ, Weston M. The effects of same-session combined exercise training on cardiorespiratory and functional fitness in older adults: a systematic review and meta-analysis. Aging Clinical and Experimental Research. 2019.

37. Hwang PW, Braun KL. The Effectiveness of Dance Interventions to Improve Older Adults' Health: A Systematic Literature Review. Altern Ther Health Med. 2015;21(5):64-70.

38. Katsoulis K, Stathokostas L, Amara CE. The Effects of High- Versus Low-Intensity Power Training on Muscle Power Outcomes in Healthy, Older Adults: A Systematic Review. J Aging Phys Act. 2019;27(3):422-39.

39. King A, Eitivipart C. Systematic Review of Published Research on Aquatic Exercise for Balance in the Elderly. Journal of Aquatic Physical Therapy. 2016;24(1):9-21.

40. Labott BK, Bucht H, Morat M, Morat T, Donath L. Effects of Exercise Training on Handgrip Strength in Older Adults: A Meta-Analytical Review. Gerontology. 2019;65(6):686-98.

41. Meereis-Lemos ECW, Guadagnin EC, CB M. Influence of strength training and multicomponent training on the functionality of older adults: systematic review and meta-analysis.. Revista Brasileira de Cineantropometria \& Desempenho Humano. 2020;22.

42. Lesinski M, Hortobágyi T, Muehlbauer T, Gollhofer A, Granacher U. Effects of Balance Training on Balance Performance in Healthy Older Adults: A Systematic Review and Meta-analysis. Sports Medicine. 2015;45(12):1721-38.

43. Leung DP, Chan CK, Tsang HW, Tsang WW, Jones A. Tai chi as an intervention to improve balance Loading [MathJax]/jax/output/CommonHTML/fonts/TeX/fontdata.js ta-analytical review.. Alternative therapies in 
health and medicine. 2011;17(1):40-8.

44. Levin O, Netz Y, Ziv G. The beneficial effects of different types of exercise interventions on motor and cognitive functions in older age: a systematic review. Eur Rev Aging Phys Act. 2017;14:20.

45. Liberman K, Forti LN, Beyer I, Bautmans I. The effects of exercise on muscle strength, body composition, physical functioning and the inflammatory profile of older adults: a systematic review. Curr Opin Clin Nutr Metab Care. 2017;20(1):30-53.

46. Liu CJ, Chang WP, Araujo de Carvalho I, Savage KEL, Radford LW, Amuthavalli Thiyagarajan J. Effects of physical exercise in older adults with reduced physical capacity: meta-analysis of resistance exercise and multimodal exercise. Int J Rehabil Res. 2017;40(4):303-14.

47. Liu H, A. F. Tai chi as a balance improvement exercise for older adults: a systematic review. J Geriatr Phys Ther. 2010;33(3):103-9.

48. Liu X, Shen PL, Tsai YS. Dance intervention effects on physical function in healthy older adults: a systematic review and meta-analysis. Aging Clin Exp Res. 2020;33(2):253-63.

49. Martin JT, Wolf A, Moore JL, Rolenz E, DiNinno A, Reneker JC. The effectiveness of physical therapist-administered group-based exercise on fall prevention: a systematic review of randomized controlled trials. J Geriatr Phys Ther. 2013;36(4):182-93.

50. Martins AC, Santos C, Silva C, Baltazar D, Moreira J, Tavares N. Does modified Otago Exercise Program improves balance in older people? A systematic review. Prev Med Rep. 2018;11:231-9.

51. Montero D, Diaz-Canestro C. Endurance training and maximal oxygen consumption with ageing: Role of maximal cardiac output and oxygen extraction. Eur J Prev Cardiol. 2016;23(7):733-43.

52. Moore M, Warburton J, O'Halloran PD, Shields N, Kingsley M. Effective Community-Based Physical Activity Interventions for Older Adults Living in Rural and Regional Areas: A Systematic Review. J Aging Phys Act. 2016;24(1):158-67.

53. Moran J, Ramirez-Campillo R, Granacher U. Effects of Jumping Exercise on Muscular Power in Older Adults: A Meta-Analysis. Sports Med. 2018;48(12):2843-57.

54. Plummer P, Zukowski LA, Giuliani C, Hall AM, Zurakowski D. Effects of Physical Exercise Interventions on Gait-Related Dual-Task Interference in Older Adults: A Systematic Review and MetaAnalysis. Gerontology. 2015;62(1):94-117.

55. Qi M, Moyle W, Jones C, Weeks B. Tai Chi Combined With Resistance Training for Adults Aged 50 Years and Older: A Systematic Review. J Geriatr Phys Ther. 2020;43(1):32-41.

56. Raymond MJ, Bramley-Tzerefos RE, Jeffs KJ, Winter A, Holland AE. Systematic review of highintensity progressive resistance strength training of the lower limb compared with other intensities of strength training in older adults. Arch Phys Med Rehabil. 2013;94(8):1458-72.

57. Rodrigues-Krause J, Krause M, Reischak-Oliveira A. Dancing for Healthy Aging: Functional and Metabolic Perspectives. Altern Ther Health Med. 2019;25(1):44-63.

58. Roland KP, Jakobi JM, GR. J. Does yoga engender fitness in older adults? A critical review. J Aging Phys Act 2011;19(1):62-79. 
59. Sivaramakrishnan D, Fitzsimons C, Kelly P, Ludwig K, Mutrie N, Saunders DH, et al. The effects of yoga compared to active and inactive controls on physical function and health related quality of life in older adults- systematic review and meta-analysis of randomised controlled trials. Int $\mathrm{J}$ Behav Nutr Phys Act. 2019;16(1):33.

60. Stathokostas L, Little RM, Vandervoort AA, Paterson DH. Flexibility training and functional ability in older adults: a systematic review. J Aging Res. 2012;2012:306818.

61. Straight CR, Lindheimer JB, Brady AO, Dishman RK, Evans EM. Effects of Resistance Training on Lower-Extremity Muscle Power in Middle-Aged and Older Adults: A Systematic Review and MetaAnalysis of Randomized Controlled Trials. Sports Med. 2016;46(3):353-64.

62. Tschopp M, Sattelmayer MK, Hilfiker R. Is power training or conventional resistance training better for function in elderly persons? A meta-analysis. Age Ageing. 2011;40(5):549-56.

63. Van Abbema R, De Greef M, Craje C, Krijnen W, Hobbelen H, Van Der Schans C. What type, or combination of exercise can improve preferred gait speed in older adults? A meta-analysis. BMC Geriatr. 2015;15:72.

64. Vetrovsky T, Steffl M, Stastny P, Tufano JJ. The Efficacy and Safety of Lower-Limb Plyometric Training in Older Adults: A Systematic Review. Sports Medicine. 2019;49(1):113-31.

65. Waller B, Ogonowska-Słodownik A, Vitor M, Rodionova K, Lambeck J, Heinonen A, et al. The effect of aquatic exercise on physical functioning in the older adult: a systematic review with meta-analysis. Age and Ageing. 2016;45(5):593-601.

66. Yang Y-P, Lin H-C, Chen K-M. Functional Fitness in Older Adults. Topics in Geriatric Rehabilitation. 2019;35(4):238-47.

67. Stares A, Bains M. The Additive Effects of Creatine Supplementation and Exercise Training in an Aging Population: A Systematic Review of Randomized Controlled Trials. J Geriatr Phys Ther. 2020;43(2):99-112.

68. Antoniak AE, Greig CA. The effect of combined resistance exercise training and vitamin D3 supplementation on musculoskeletal health and function in older adults: a systematic review and meta-analysis. BMJ Open. 2017;7(7):e014619.

69. Gade J, Pedersen RJ, AM. B. Effect of Protein or Essential Amino Acid Supplementation During Prolonged Resistance Exercise Training in Older Adults on Body Composition, Muscle Strength, and Physical Performance Parameters: A Systematic Review. Rehabilitation Process and Outcome. 2018;7(7):1-12.

70. Hanach NI, McCullough F, Avery A. The Impact of Dairy Protein Intake on Muscle Mass, Muscle Strength, and Physical Performance in Middle-Aged to Older Adults with or without Existing Sarcopenia: A Systematic Review and Meta-Analysis. Adv Nutr. 2019;10(1):59-69.

71. Devries MC, Phillips SM. Creatine supplementation during resistance training in older adults-a metaanalysis. Med Sci Sports Exerc. 2014;46(6):1194-203.

72. Hou L, Lei Y, Li X, Huo C, Jia X, Yang J, et al. Effect of Protein Supplementation Combined with 
Meta-Analysis. J Nutr Health Aging. 2019;23(5):451-8.

73. Ten Haaf DSM, Nuijten MAH, Maessen MFH, Horstman AMH, Eijsvogels TMH, Hopman MTE. Effects of protein supplementation on lean body mass, muscle strength, and physical performance in nonfrail community-dwelling older adults: a systematic review and meta-analysis. Am J Clin Nutr. 2018;108(5):1043-59.

74. Finger D, Goltz FR, Umpierre D, Meyer E, Rosa LH, Schneider CD. Effects of protein supplementation in older adults undergoing resistance training: a systematic review and meta-analysis. Sports Med. 2015;45(2):245-55.

75. Meereis-Lemos ECW, Guadagnin EC, CB M. Influence of strength training and multicomponent training on the functionality of older adults: systematic review and meta-analysis. Rev Bras de Cineantropometria \& Desempenho Humano. 2020;22(e60707).

76. Stathokostas L, Little RM, Vandervoort AA, Paterson DH. Flexibility training and functional ability in older adults: a systematic review. J Aging Res. 2012;2012(306818):1-13.

77. Hurst C, Weston KL, McLaren SJ, Weston M. The effects of same-session combined exercise training on cardiorespiratory and functional fitness in older adults: a systematic review and meta-analysis. Aging Clinical and Experimental Research. 2019;31(12):1701-17.

78. McLaughlin EC, El-Kotob R, Chaput JP, Janssen I, Kho ME, Poitras VJ, et al. Balance and functional training and health in adults: an overview of systematic reviews. Appl Physiol Nutr Metab. 2020;45(10 (Suppl. 2)):S180-S96.

79. El-Kotob R, Ponzano M, Chaput JP, Janssen I, Kho ME, Poitras VJ, et al. Resistance training and health in adults: an overview of systematic reviews. Appl Physiol Nutr Metab. 2020;45(10 (Suppl. 2)):S165-S79.

80. Ross R, Chaput JP, Giangregorio LM, Janssen I, Saunders TJ, Kho ME, et al. Canadian 24-Hour Movement Guidelines for Adults aged 18-64 years and Adults aged 65 years or older: an integration of physical activity, sedentary behaviour, and sleep. Appl Physiol Nutr Metab. 2020;45(10 (Suppl. 2)):S57-S102.

81. Burton E, Farrier K, Lewin G, Pettigrew S, Hill AM, Airey P, et al. Motivators and Barriers for Older People Participating in Resistance Training: A Systematic Review. J Aging Phys Act. 2017;25(2):311-24.

82. Devereux-Fitzgerald A, Powell R, Dewhurst A, French DP. The acceptability of physical activity interventions to older adults: A systematic review and meta-synthesis. Soc Sci Med. 2016;158:1423.

\section{Table 2}

Due to technical limitations, table 2 is only available as a download in the supplementary files section.

\section{Fiaures}




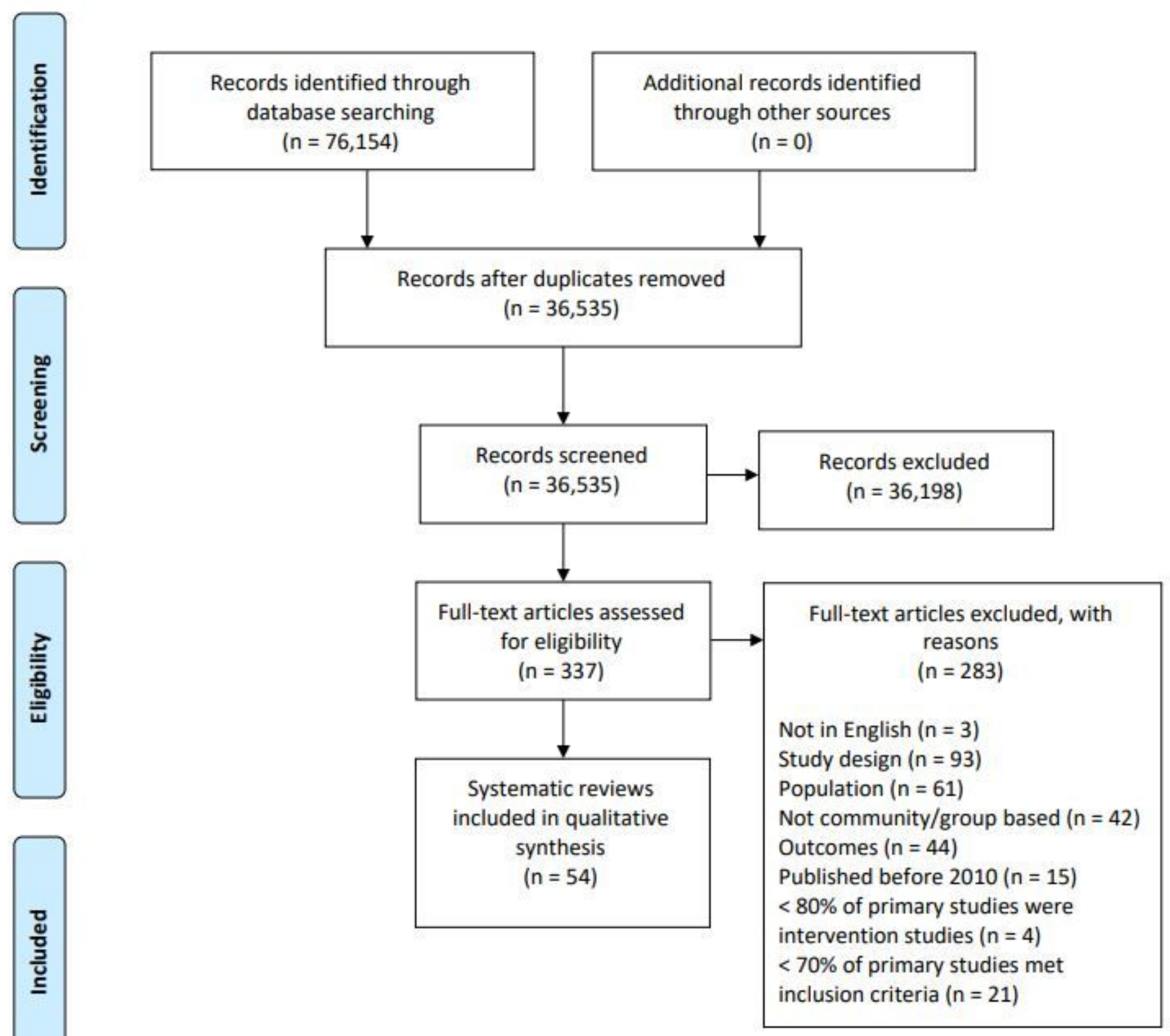

Figure 1

PRISMA Flow Diagram 
Protocol

Study design

Search strategy

Duplicate selection

Duplicate extraction

List excluded studies

Describe included stdies

Risk of bias - RCTs

Risk of bias - NRSI

Sources of funding

Appropriate statistical methods - RCTs

Appropriate statistical methods - NRSI

Risk of bias, meta-analysis

Incorporate risk of bias

Heterogeneity

Publication bias

Conflict of interest

$0 \%$

Legend: $\mathrm{RCT}$ = randomized controlled trial, $\mathrm{NRSI}=$ non-randomized studies of interventions

-Yes Partial yes $=$ No

\section{Figure 2}

AMSTAR 2 Summary of Systematic Review Quality

\begin{tabular}{|c|c|c|c|c|c|}
\hline & Aerobic capacity & Physical function & Balance & Falls/safety & Muscular strength \\
\hline Resistance & $\leftrightarrow$ very low certainty ${ }^{a, c, c}$ & $\uparrow$ low certainty ${ }^{\text {h. }}$ & $\leftrightarrow$ low certainty b,e & $\leftrightarrow$ Very low certainty $\mathrm{b}, c, c$ & $\uparrow$ moderate certainty \\
\hline Aerobic & $\uparrow$ moderate certainty & $\leftrightarrow$ low certainty ${ }^{\natural, c}$ & $\leftrightarrow$ low certainty b,c. & $\leftrightarrow$ very low certainty ${ }^{\mathrm{b}, c, e}$ & $\uparrow$ low certainty $k c c$ \\
\hline Aerobic + Resistance & $\uparrow$ high certainty & $\uparrow$ moderate certainty ${ }^{2}$ & $\uparrow$ moderate certainty ${ }^{c}$ & $\uparrow$ moderate certainty ${ }^{c}$ & $\uparrow$ low certainty $h c$ \\
\hline General physical activity & $n / a$ & $\uparrow$ moderate certainty ${ }^{c}$ & $\uparrow$ moderate certainty ${ }^{c}$ & $\uparrow$ moderate certainty ${ }^{c}$ & $\uparrow$ moderate certainty ${ }^{c}$ \\
\hline Exercise and nutrition & $\leftrightarrow$ low certainty ${ }^{\mathrm{d}, c}$ & $\leftrightarrow$ low certainty ${ }^{\mathrm{b}, \mathrm{E}}$ & $n / a$ & $n / a$ & $\leftrightarrow$ low certainty ${ }^{\mathrm{B}, \mathrm{c}}$ \\
\hline Mind-body exercise & $\leftrightarrow$ very low certainty ${ }^{c, d, e}$ & $\uparrow$ moderate certainty $=$ & $\uparrow$ high certainty & $\leftrightarrow$ low certainty ${ }^{c, c}$ & $\uparrow$ low certainty ${ }^{h=}$ \\
\hline Dance & $\uparrow$ low certainty ${ }^{b, c}$ & $\uparrow$ low certainty ${ }^{\text {h.c }}$ & $\uparrow$ low certainty ${ }^{b, c}$ & $\uparrow$ very low certainty ${ }^{b, d, c}$ & $\uparrow_{\text {low certainty }}{ }^{h, c, 2}$ \\
\hline \multicolumn{2}{|c|}{$\begin{array}{l}\text { Notes: } \\
\text { s start at low certainty due to non-randomized study designs }\end{array}$} & \multicolumn{4}{|c|}{$\begin{array}{l}\text { 2 start at low certainty due to non-randomized study designs } \\
\text { s downgraded due to risk of bias }\end{array}$} \\
\hline \multicolumn{6}{|c|}{$\begin{array}{l}\text { 'downgraded due to inconsistency in effects } \\
\text { A downgraded due to indirectness of interventions'outcomes }\end{array}$} \\
\hline \multicolumn{6}{|c|}{ - downgraded due to imprecision in effect estimate } \\
\hline $\begin{array}{l}\text { upgraded due to large eff } \\
\text { upgraded due to dose-res } \\
\text { upgraded due to accounti }\end{array}$ & $\begin{array}{l}\text { nship } \\
\text { Inding }\end{array}$ & & & & \\
\hline
\end{tabular}


Figure 3

GRADE Summary of Certainty of Evidence

\section{Supplementary Files}

This is a list of supplementary files associated with this preprint. Click to download.

- table2.docx 